\title{
"What are We Waiting for, Another Child to Die?" A Qualitative Analysis of Regulatory School Environments for Food Allergic Children
}

\author{
Susan J Elliott ${ }^{1, *}$, Nancy Fenton ${ }^{2}$, C-L Joanna Sinn ${ }^{2}$,Ann Clarke ${ }^{3}$ \\ ${ }^{1}$ Department of Geography \& Environmental Management, University of Waterloo, Canada \\ ${ }^{2}$ School of Public Health and Health Systems, University of Waterloo, Canada \\ ${ }^{3}$ Department of Medicine, University of Calgary, Canada
}

Copyright $(C 2015$ by authors, all rights reserved. Authors agree that this article remains permanently open access under the terms of the Creative Commons Attribution License 4.0 International License

\begin{abstract}
Severe food allergy, or anaphylaxis, is an immediate and severe allergic reaction that may cause difficulty breathing, hypotension, unconsciousness, and even death. Given there is no cure, managing life-threatening reactions is limited to strict avoidance of implicated foods and provision of medication for symptomatic treatment of an adverse reaction. A key piece of this management is the regulatory frameworks used in public spaces such as schools. This paper explores the perceptions and experiences of 'school' as a safe place for students with anaphylaxis and their parents in two Canadian provinces with very different regulatory environments in order to assess the impact of alternative regulatory environments on the quality of life of affected individuals. A qualitative research design involving draw-and-tell exercises along with in-depth interviews with elementary school-aged children $(n=45)$ and their parents in the provinces of Ontario and Quebec, Canada was used. Results indicate that these very different regulatory environments had differing impacts on kids and parents. Ontario parents found security in the Ontario legislation, while the heterogeneity of the Quebec school regulatory environments resulted in reported stress and anxiety among the parents interviewed. The kids themselves just wanted to feel normal and not be teased or bullied.
\end{abstract}

Keywords Anaphylaxis, School Environment, Regulatory Frameworks, Qualitative Analysis, Canada

\section{Introduction}

A recent op-ed piece in Canada's national newspaper The Globe \& Mail - pointed to a regulatory crisis in Canadian schools for children with chronic illness, including anaphylactic food allergy (1). Anaphylaxis is an immediate and severe allergic reaction that may cause difficulty breathing, hypotension, unconsciousness, and even death. Onset of life-threatening allergies in children often occurs in infancy and early childhood, affecting approximately $8 \%$ of US children [2, 3] and approximately $7.5 \%$ of Canadian children [4]. Recent Canadian data indicate that fully $50 \%$ of households are affected, either directly or indirectly, by food allergy [5]. Given the above, families have to learn to cope early in life with the risks of exposure to danger and even death.

Given there is no cure, managing life-threatening reactions is limited to strict avoidance of implicated foods and provision of medication (i.e., epinephrine auto-injectors, or EpiPens) for symptomatic treatment of an adverse reaction $[6,7]$. Avoidance of specific food allergens means food allergic children, their families, and others with whom they come in contact (e.g., friends, relatives, school personnel) must remain vigilant against the constant threat of exposure [8, 9]. Regardless of parents' best efforts to maintain control and safety at home, children are often exposed to unfamiliar people, places, and routines particularly at the time they start school. School environments can be fraught with risks (e.g., lack of awareness, bullying) that trigger anxiety and stress and impact the quality of life of food allergic children and their parents $[5,10]$.

In addressing this situation, some schools provide internal controls geared toward providing safe environments. This may involve steps along a continuum from awareness raising to the banning of particular foods (e.g., peanut). In other cases, school board and/or regional (e.g., Provincial) policies protect food allergic children in the school environment. A more extreme example comes from the province of Ontario, Canada where the assurance of safety for food allergic children in the school environment is provided for under Bill 3: An Act to Protect Anaphylactic Pupils. This paper explores the perceptions and experiences of 'school' as a safe place for students with anaphylaxis and their parents in 
two Canadian provinces with very different regulatory environments in order to assess the impact alternative regulatory environments might have on the quality of life of affected individuals.

Intuitively, it makes sense that dealing with any health condition can affect one's quality of life. In this instance, added weight comes from the age (young children) and vulnerability (life threatening) associated with food related anaphylaxis. For example, Sicherer et al. [11] found that food allergic children and adolescents reported significantly poorer results than their healthy counterparts for general health perception and parental perceptions of distress and worry. Somewhat surprisingly, one study showed that quality of life was more greatly impacted in children with food allergy than children with insulin-dependent diabetes mellitus, with food allergic children reporting more fear, anxiety, and restrictions than their diabetic counterparts [12]. Indeed, there is a rich literature describing the health related quality of life of food allergic children that need not be further summarized here [see, for example, 13- 16, 9]. What is absent from this literature, however, is an assessment of the impact of the regulatory environment on the quality of life of affected individuals; this is somewhat surprising given that active avoidance remains the only valid treatment option for food allergic children and those close to them.

An Act to Protect Anaphylactic Pupils (i.e., Sabrina's Law) came into effect in Ontario in January 2006 - three years after the death of 13-year-old Sabrina Shannon in her school cafeteria. Sabrina died of an anaphylactic reaction to a dairy allergy. Her EpiPen was in her school locker, down the hall. She was a very well trained anaphylactic child who asked all the right questions; what killed her was cross contamination of serving utensils.

In essence, Sabrina's Law law requires that all publically funded school boards in Ontario ensure that schools put in place measures to safeguard students at risk of anaphylaxis including: exposure risk reduction strategies in classrooms and common school areas; communication plans to parents, students, and school personnel; regular staff training including proper administration of the Epipen; individualized student plans including current treatment information, a readily accessible emergency procedure, and storage for epinephrine auto-injectors (EpiPens); and a requirement to declare life-threatening allergies upon school registration (An Act to Protect Anaphylactic Pupils (Sabrina's Law), 2005, S.O. 2005, C.7, $<\mathrm{http}: / /$ www.e-laws.gov.on.ca/html/statutes/english/elaws_s tatutes_05s07_e.htm $>$ retrieved on 2015-07-22). Additionally, Sabrina's Law grants immunity to any person who acts in good faith in response to an anaphylactic reaction.

Unlike Ontario, the Province of Quebec presently has no province-wide anaphylactic policy let alone enacted legislation that would serve to unify individual school protocols. Each school board and regional child-care centre develops its own protocol outlining staff responsibilities and treatment guidelines. There are many similarities across protocols employed throughout the Province - for example, most schools require storage for EpiPens and some schools have banned peanut and/or nut products completely. School nurses have the primary responsibility to complete an emergency plan for each student, and they are given training and resources by the Association Québécoise des Allergies Alimentaires (AQAA) (Quebec's only referral centre for food allergies).

Sabrina's Law in Ontario and individual school protocols in Quebec both aim to provide a safe environment for food allergic schools in their respective provinces; however, there is some evidence that the heterogeneity of guidelines places food allergic children at additional risk. For example, Cicutto et al. [17] compared school anaphylaxis management practices in Canadian schools with legislation (Ontario) and without legislation (Alberta, British Columbia, Newfoundland and Labrador, and Quebec). The authors found that parents in a legislated environment were more likely to report that children's schools were supportive of their food allergic children while parents in a non-legislated environment were more likely to report that the lack of awareness and staff training in schools had placed their child at risk. This paper extends Cicutto's work by exploring the lived experiences of food allergic children, and their parents, with respect to the provision of a safe school environment. Specifically, the aim of this research is to explore the perceptions and experiences of 'school' as a safe place for anaphylactic students and their parents in two distinct Canadian policy environments, in order to understand the impact of the regulatory environment on risk perception, risk response, and quality of life.

\section{Materials and Methods}

This study employed an innovative qualitative research design. While the lion's share of existing literature around food allergy is quantitative in nature, there is increasing recognition of the importance and the inherent value of qualitative epistemologies:

Qualitative research has enormous potential to make a distinctive contribution to knowledge about allergy management and to provide insights that quantitative studies cannot. In particular, qualitative research can afford understanding of patients' perspectives and illuminate the often-neglected personal and social contexts of illness and care...This is especially important in the case of people experiencing allergic problems, where management generally involves long-term strategies carried out by patients themselves. Yet qualitative research is rarely reported in allergy journals [18:1117]

In undertaking this research, we employed draw-and-tell exercises as well as in-depth interviews with elementary school-aged children $(\mathrm{n}=45)$ and their parents. The Ontario sample was recruited from a registry maintained by 
Anaphylaxis Canada (a national non-profit organization that supports families with members at risk for anaphylaxis) using newsletter announcements, direct email, and targeted bulletins. The Quebec sample was recruited from a registry of families with clinically diagnosed peanut allergic children in the Montreal area. The sample was balanced for gender. A University Research Ethics Board approved the study.

Parent and child interviews were conducted separately by the same interviewer. The Quebec interviews were conducted in either English or French. Parents were asked about their experiences of sending their children to school and their feelings about school as a safe place. They were also asked about their views on the food allergy policies in place (or lack thereof) in their child's school. Children were asked to draw a picture of "what it is like to live with a food allergy' and then to explain their illustration. This draw-and-tell exercise facilitated an in-depth interview that explored more fully their perceptions and experiences of coping with food allergies at school.

Interviews, averaging 60 minutes in length, were audio-recorded, transcribed verbatim, and member-checked with participants for subsequent thematic analysis using NVIVO. Results are organized around key themes emerging from the transcripts related to the positive and negative life experiences of coping with food allergies in schools, for both kids and parents.

\section{Results}

\subsection{Positive Emotional Contexts and Coping Strategies for Kids}

The majority of children in both Ontario and Quebec recalled positive social and emotional experiences occurring in their school. Having an understanding group of friends and feeling normal emerged as common mediators of these positive experiences. To these kids, having an 'understanding group of friends' meant: friends were fully aware of the consequences of coming into contact with food allergen(s) and ensured that any food items they brought to school could not hurt their food allergic friend:

Yea, they understand like, they don't understand my habits and stuff that I do to cope with it. They understand that I have it really, but that is about it; that you have to read the label before I eat it. [Ontario, child]

Sometimes their friends even acted as advocates on their behalf:

Because, as I was saying, my friends tell other people that I'm allergic and they also tell them, "Don't tease him or anything." [Quebec, child]

Having a sense of being a 'normal' student - not being different from others - was a second mediator of positive social and emotional experiences at school. When asked about their day-to-day school routine, food allergic children with positive experiences did not see any differences between their routines and the routines of their non-food allergic friends. They viewed their allergies as only a minor and usually hidden part of their self that did not change their school lives or their friendships.

I am just like every other kid. I go to school. No special treatment. I am just a regular kid, only like I don't get to have certain foods. I don't learn anything different. I don't go out to classes with other teachers. [Ontario, child]

Defining normal differed across regulatory environments. While in Ontario, kids did not want to be treated differently, Quebec kids often defined normal through the routines established around their food allergy. For example, in some Quebec schools, food allergic children ate lunch all together at a separate allergy table. Indeed, some children felt more 'normal' among their food allergic peers even if they were segregated from their non-food allergic peers. In this way, they too also had their understanding group of friends.

I don't think he feels abnormal. I don't think he feels, you know, that it's really weird or strange. And the fact that they put all of the allergic children together at the school, also, I think they feel like, you know, just part of the school community. They just can't eat everything that other people can eat. [Quebec, parent]

A primary coping strategy for children from both provinces was maintaining open relationships with classmates and teachers. Most felt confident in communicating their allergy needs as appropriate - for example, when making a new friend or when recognizing a potential exposure.

Well, if you have a food allergy, then it sort of can help because you learn how to talk to them. Like if you do want to make a new friend, you learn how to talk to them...you won't just say, "Hi, what is your name?" You will say, "Hi, my name is whatever and I have a food allergy." And you will learn how to say that to people when [you] do make new friends. [Ontario, child]

Early in the year, his teacher came in eating a piece of toast and he went up to her and he said, "Did you just have a peanut butter toast?" And she said, "Oh my goodness, you're right. I did." and he asked her politely, "Could you, when you're finished, could you please go wash your hands?" [Quebec, parent]

In the context of anaphylaxis management strategies in place at school that helped them to cope and to feel safe at school, both Ontario and Quebec students had lots to say. Nearly all Quebec children talked about knowledgeable staff and students who knew the location of and how to use their EpiPen.

I would just like run [if I started to react] and take out my EpiPen. Then my friends will know this because they're always around me because they know how to 
use it because I showed them a little bit like how to do it. Like with a pen I did. And they would take it, do the thing and do it. [Quebec, child]

In contrast, Ontario children more frequently cited complementary strategies in addition to keeping EpiPens on themselves or in the school.

Yes, I feel fairly safe at school. I know I have my EpiPens most of the time, and I have pens in the drawers. I know my whole class knows about it, whether they paid attention or not to the things. I know they know... And people aren't allowed to bring peanut snacks at school, and some of the kids, they do read the labels. [Ontario, child]

\subsection{Negative Emotional Contexts and Coping Strategies for Kids}

Food allergic children in both provinces reported negative school experiences potentially linked to higher levels of stress and anxiety. Even though friends often provided safety nets and acted as key supports, many children still encountered difficulty when dealing with uninformed people (e.g., students, teachers, other school personnel) and the impacts of social exclusion and teasing. These kids worried about making new and/or maintaining current friendships because, for example, their food allergy necessarily impacts the food choices of others, as well as the structure of food consumption:

Well, school itself is fine, but she gets a little irritated sometimes with other students because they kind of bug her in a way, you know, "Oh, the peanut allergy girl." [Quebec, parent]

Like usually, I am not sitting in the cafeteria with everyone else, like I might not be as talkative with them in that period of time. So then, like, they could be talking about something in the cafeteria and then come outside and keep talking about it, and I won't be able to join in on the conversation... I don't know. Like sometimes I feel like I am losing friends. [Ontario, child]

The immediate consequences of rejection or feeling different are clear; however, these negative experiences may continue to threaten the food allergic child's social well-being. Some parents were concerned that their children's trust of new people and new places has been permanently affected:

And now he just, he wants to get involved, but he doesn't want to. He wants to stay with the people that he knows. Like, he doesn't want any new type of friends, so to speak, because they don't know his situation. [Quebec, parent]

Kids are also constantly worried about coming into contact with food allergen(s) from other children and/or the broader school environment. Snack and lunch times were always associated with higher anxiety, but birthday celebrations were particularly stressful. Often comparing school to the security of home, they felt stressed about negotiating new patterns, encountering unsafe foods, and dealing with uninformed people. They felt that others did not place enough importance on maintaining allergen-free environments; thus, these children compensated by redoubling their efforts to be constantly vigilant against the risk of exposure.

I have a certain cautiousness to what I do, and like every time I want to eat something, I have to double check it. So that if somebody brings in snacks to school or something like, I am constantly on the lookout for something. I feel uptight and kind of scared about some stuff. [Ontario, child]

And people, like, they tease me a lot. When I tell them that they have to wash their hands or the teacher does, then they go like this [hand action]. They just like to scare me. [Quebec, child]

\subsection{Positive Emotional Contexts and Coping Strategies for Parents}

Parents' comments echoed those of the kids regarding the dependability of their friendships.

Kids have a lot of respect for each other when it comes to that. It's very touching and striking. There's a little girl that comes here and she tells me, "I don't want to eat peanut butter in the morning because I don't want [your child] to be sick." If only parents could be half like that, it would be great. [Quebec, parent]

Yet positive stories generally emerged from the existence of a champion in the school, often a particular teacher or principal:

I think we have been very lucky. They have been very good, very supportive. The teachers have always been... We have always met. We have contacted them at the beginning of the year, just to remind them about it, and they usually already know now... They have it covered. [Ontario, parent]

His teacher's great...we communicate all the time by phone and so she's very conscious and I'm very careful and so far there hasn't been any problems or any accidents at school. [Quebec, parent]

Beyond these very personal relationships, all Ontario parents believed standards needed to be legislated in order to provide consistent and accountable procedures to ensure safety. Ontario parents frequently cited Sabrina's Law as a source of additional confidence and support - beyond depending on local champions for the safety of their food allergic children.

I think our school has always been good, but it [Sabrina's Law] has given us just a 
little more confidence to say, "I know you have to provide this to me." [Ontario, parent]

I think [the school] could have [implemented these strategies without Sabrina's Law], but it would depend on who was there, and I think that is too dangerous to just take that sort of roulette wheel. [Ontario, parent]

For Ontario parents, Sabrina's Law also appeared to modulate the degree of parental involvement in advocacy and educational activities. Several parents were fully involved in ensuring a safe school environment for their children through activities such as putting up posters, being in constant communication with staff, providing educational instruction to staff on EpiPen use, and sitting on parent council.

I have been very involved in a lot of allergy awareness at our school, and I am kind of the go to person. When they have a new person come to the school, the principal usually calls me and says, "We have a new person, can you talk to them about it? Or they don't have an EpiPen or they don't have an EpiPen holder. Can you tell them?" [Ontario, parent]

However, there is some evidence that the type and degree of parental involvement has shifted as a result of Sabrina's Law, with an evident reduction in the perceived need for Ontario parents to take part in hands on advocacy. Some Ontario parents talked about spending more time on education (instead of advocacy) and less time overall regarding school policies for anaphylaxis management as a result of the new legislation.

When Sabrina's Law came in, it was a lot easier and a lot better. I didn't have to go in full force with everything because there were already guidelines that they were supposed to use. [Ontario, parent]

I can remember, you know, the summer before he started, thinking, "I will write a note to all the parents." I never did it, because as time got closer, I realized he is going to be fine. [Ontario, parent]

Quebec parents, on the other hand, appeared to be much more hands on in the school setting, and remained reliant on strong communication and local champions.

\subsection{Negative Emotional Contexts and Coping Strategies of Parents}

Although Ontario parents more frequently recalled positive emotional contexts than their Quebec counterparts, some Ontario parents were still highly anxious about their child in the school environment. For both groups of parents, stress escalated when parents lost control such as when children gained increased independence when starting school or transitioned to new school environments.

You just feel like control is taken from you. It made me worried when she was out of my control, or out of my environment. How was she going to be safe? Sending her to school was terrifying. [Ontario, parent]

Needing to have my phone with me all the time... especially during school hours. She is at school, or she is away somewhere or something. It used to bother me if my phone used to ring. I would feel panicked. [Ontario, parent]

However, in the absence of a legislated environment like Ontario's, a number of Quebec parents felt threatened by the variability and inconsistency of school policies. Some parents described the "battles" of coping with uninformed school personnel and inaccessible EpiPens in their child's school, both present and future (i.e., transitioning to middle and high school).

It's the teacher's call. It's not the school. The school will not take a stand. [Quebec, parent]

But it's a scary thing though, because you know, the awareness is there now but then when I leave the school and then this other mother leaves the school. I don't know, if they're okay. Okay, they're going to be still aware but I don't know. I don't know - I just think it's, there's no consistency. I don't think it's strict enough. I really don't. [Quebec, parent]

Some Quebec parents believed that implementing standard anaphylaxis management policies across school boards - as required by Sabrina's Law in Ontario - was the solution to creating safer schools for all food allergic children.

... my sister-in-law works at a private school in Ontario and she said they're so, like they have an emergency plan for every child. They have training - how to use the EpiPen. They've got like everything in place. Like they're ready. Like it's unbelievable. They're trained to the max. And it's law. It's the law. But here it's kind of wishy-washy; some schools are good, some schools aren't good. And it just doesn't make sense to me. They're just taking too much risk. [Quebec , parent]

It should be [Sabrina's Law]. Yeah, I think that it really has to. I'm stunned that it hasn't come to that yet. I'm really - I don't know - like what are they waiting for? For another child to die in Quebec? I mean it's stunning. [Quebec, parent]

This blanket solution, however, was not a sentiment shared by all Quebec parents. In fact, some parents believed that food allergies should remain an individual responsibility that lies outside of the realm of provincial legislation or even school policy.

They were on the committee and my husband was actually against making the school peanut-free and parents sort of looked at him and they go, "Well you don't have a child with allergies. You just don't understand." And we found out that [our child] had allergies, like peanut allergies. We said, "Well now we 
understand and we're still against the policy." Because I find when you make a school peanut-free, people let their guard down and they're not as careful. And I think it's my daughter's responsibility and my responsibility to be careful and to make sure that people around her know... And you can't control all those people and all those families. [Quebec, parent]

In Quebec, some parents retreated from advocacy activities while other parents internalized their food allergy "problem" completely. Instead, parents focused their energy on teaching their child to practise constant vigilance.

You know I think what's my role going to be? I think it's to make parents aware that, you know, you can only expect so much from the school. [Quebec, parent]

Unless you live with the allergy, you're not obsessive about looking for, at the ingredients. So, you know, I never asked at the school that it be peanut-free. I know it's impossible for it to be peanut-free. I'd rather he, you know, he learns. Like if he goes out, he puts a napkin on the table or in case of crumbs. You know, he has to learn to live in a peanut world. [Quebec, parent]

While there was no clear majority among Quebec parents regarding direction for school anaphylaxis management policies, inconsistent school policies led parents of food allergic children to choose schools based on size, sensitivity of staff, and/or comprehensiveness of existing policies and/or practices. This unintended consequence may emerge through the greater number of food allergic children enrolled in private or smaller-sized schools. Parents reported feeling that a private school allows for more frequent communication with school personnel, and that a smaller school increases the likelihood that the whole school community knows about their child's allergy.

Well yes [we can get in touch with the teachers and administration]. That's why he's in a private school. Because of his allergies, I wanted him to be well supervised... Also a private school tends to be smaller; it's 600 pupils, not a thousand. [Quebec, parent]

That's one of the reasons we sent him there, because it's a smaller school and they're very sensitive to allergies. And we just felt more comfortable that way, as opposed to sending him to one of these enormous schools where he's lost in the crowd and nobody really knows. [Quebec, parent]

\section{Discussion}

There is concern that food allergies have been and continue to increase across North America and beyond [19, 20]. Regardless, all these young children with food allergies will continue to grow into young adulthood and, as a society, we will have to determine how best to create regulatory environments to ensure feelings of safety for affected individuals, in places of post-secondary education, the work place, and beyond. This small qualitative exploration of regulatory environments begins to point us in a direction that may shed light on these issues.

The results presented here illustrate an interesting dynamic around the regulatory school environment in the face of managing life threatening food allergies for children. In some cases, the grass appears to be greener on the other side, with some Quebec parents coveting the Ontario legislation, while others continue to report the importance of individual responsibility. This is consistent with the literature [21] that some of the most common reasons for opposing a food allergy-related regulation are related to the impossibility of making a school 'allergen free', that food bans provide a false sense of security, the difficulty inherent in enforcing food bans, and undue hardship on the broader school community.

While the efforts of these Quebec parents occasionally re-shaped unsafe and exclusionary practices at individual schools, a lingering uncertainty prevailed because of the reliance on individual effort and resources for the tenuous enforcement of policies. For many parents, the "fight" to keep their children safe at school sustained high levels of anxiety and vigilance that placed greater burden on them. Indeed, what appears to cause the greatest anxiety for the Quebec parents is the heterogeneity of policies and procedures across school environments that in turn affect school choice and bind these parents to direct involvement in their children's daily lives vis-à-vis the school environment.

While the Ontario parents appear to appreciate the reassurance of the legislation in their Province - knowing schools MUST act, and that there are legal consequences if they do not - this seems to have affected their level of hands on involvement in the school proper. This in turn may impact on the quality of life/feelings of safety for their children, who see their parents taking less of an active role in education and/or advocacy in their school environment. This result warrants further research.

\section{Conclusions}

For the food allergic children in this study, it all comes down to feelings of normal. This is consistent with the desires of every young child, in the context of their peer group. That is, at best, allergic kids do not want to be seen as different and at worst they do not want to be teased or bullied. How can this best be accomplished through a regulatory framework? There does not appear, from the voices of these children, to be a one size fits all answer; some report feeling normal when integrated with their school peers, others report feeling normal when segregated with their food allergic peers. Some feel normal when their friends know about and are respectful of their allergy, others do not want to be 'outted'. Canada's premier health journalist, Andre Picard, in a recent op-ed piece entitled: Why do we need laws to enforce common sense? states:

Why, time and time again, does it take a dead child before 
our schools act sensibly? [1: A15].

The question remains, what is the way forward? Perhaps we should ask the children.

\section{Acknowledgements}

This work was funded by the AllerGen National Centres of Excellence (http://www.allergen-nce.ca).

\section{REFERENCES}

[1] Picard A. (2015) Why do we need laws to enforce common sense? The Globe and Mail May 5: A15.

[2] Vierk KA, Koehler KM, Fein SB, Street DA, Park C. Prevalence of self-reported food allergy in American adults and use of food labels. $J$ Allergy Clin Immunol. 2006;119(6):1504-1510.

[3] Gupta RS, Springston EE, Warrier MR, et al. The prevalence, severity, and distribution of childhood food allergy in the United States. Pediatrics. 2011;128(9):9-17.

[4] Soller L, Ben-Shoshan M, Harrington DW, et al. Overall prevalence of self-reported food allergy in Canada. J Allergy Clin Immunol. 2012;130(4):986-988.

[5] Harrington, D.W., Elliott, S.J. and Clarke A. E. (2012) Exploring the determinants of the perceived risk of food allergies in Canada. Human and Ecological Risk Assessment, 18(6):1338-1358 (Paper of the Year winner, 2012).

[6] Sampson, H. A. (2004). Update on food allergy. Journal of Allergy and Clinical Immunology, 113(5), 805-819.

[7] Sicherer, S. H. (2002). Food allergy. Lancet, 360(9334), 701-710.

[8] Cohen, B. L., Noone, S., Muñoz-Furlong, A., \& Sicherer, H. H. (2004). Development of a questionnaire to measure quality of life in families with a child with food allergy. Journal of Allergy and Clinical Immunology, 114(5), 1159-1163.

[9] Stjerna M-L., Vetander M., and Wickman M. (2013) The management of situated risk: a parental perspective on child food allergy. Health 18(2): $130-145$.

[10] Muñoz-Furlong, A. (2004). Food allergy in schools: Concerns for allergist, paediatricians parents and school staff. Annals of Allergy, Asthma \& Immunology, $93(5$ supp 3),S47-S50.
[11] Sicherer, S. H., Noone, S., \& Muñoz-Furlong, A. (2001). The impact of childhood food allergy on quality of life. Annals of Allergy, Asthma \& Immunology, 87(6), 461-464.

[12] Avery, N. J., King, R. M., Knight, S., \& Hourihane, J. O. (2003). Assessment of quality of life in children with peanut allergy. Pediatric Allergy \& Immunology, 14(5), 378-382.

[13] Matza L.S., Swensen A.R., Flood E.M., Secnik K. and Kline Leid N. (2004) Assessment of Health-Related Quality of Life in Children: A Review of Conceptual, Methodological, and Regulatory Issues. Value in Health 7(1): 79 - 92.

[14] Östblom, E. A.-C. Egmar, A. Gardulf, G. Lilja, M. Wickman (2008) The impact of food hypersensitivity reported in 9 -year-old children by their parents on health-related quality of life Allergy 63(2): 211-218.

[15] Flokstra-de Blok, B. M., DunnGalvin, A., Vieg-Boerstra, B. J., Elberink, E. J., Duiverman, E. J., Hourihane, J. O., Dubos, A. E. (2008a). Development and validation of a self-administered Food Allergy Quality of Life questionnaire for children. Clinical and Experimental Allergy, 39(1), 127-137.

[16] Flokstra-de Blok, B. M., DunnGalvin, A., Vieg-Boerstra, B. J., Elberink, E. J., Duiverman, E. J., Hourihane, J. O., Dubos, A. E. (2008b). Development and validation of a self-administered Food Allergy Quality of Life questionnaire for adolescents. Journal of Allergy and Clinical Immunology, 122(1), 139-144.

[17] Cicutto, L., Julien, B., Li, N.Y., Nguyen-Luu, N.U., Clarke, A., Elliott, S.J., Harada, L., McGhan, S., Stark, D., Vander Leek, T.K., Waserman, S. Comparing school environments with and without legislation for the prevention and management of anaphylaxis. European Journal of Allergy and Clinical Immunology, 2012, 67(1):131-137.

[18] Gallagher M., Worth A. and Sheikh A. (2009) Clinical allergy has much to gain from engagement with qualitative research. Allergy 64: 1117-1120.

[19] Sicherer S.H. and Sampson H.A. (2010) Food Allergy Journal of Allergy and Clinical Immunology, 125 (2), S2: S116-S125.

[20] Du Toit G. et al (2015) Randomized trial of peanut consumption in infants at risk for peanut allergy. New England Journal of Medicine 372:803-813.

[21] Rachul, C, Caufield T. Food allergy policy and the popular press: perspectives from Canadian newspapers. J Asthma Allergy Educ. 2011;2(6):282-287. 